\title{
THE MATTILA INTEGRAL ASSOCIATED WITH SIGN INDEFINITE MEASURES
}

\author{
Alex Iosevich And Misha Rudnev
}

August 3, 2005

\begin{abstract}
In order to quantitatively illustrate the role of positivity in the Falconer distance problem, we construct a family of sign indefinite compactly supported probability measures in $\mathbb{R}^{d}$, such that the energy of dimension $s>\frac{d}{2}$ is uniformly bounded. However, the Mattila integral (associated with the Falconer distance problem) for these measures is unbounded in the range $\frac{d}{2}<s<\frac{d^{2}}{2 d-1}$.
\end{abstract}

\section{INTRODUCTION}

Let $\mu$ be a compactly supported Borel measure in $\mathbb{R}^{d}, d \geq 2$. Suppose that $\mu$ is $s$ dimensional in the sense that its energy integral

$$
I_{s}(\mu)=\iint|x-y|^{-s} d \mu(x) d \mu(y)<\infty
$$

The question that arises often in geometric measure theory and related areas is to determine the rate of decay of the spherical average

$$
\sigma_{\mu}(t)=\int_{S^{d-1}}|\widehat{\mu}(t \omega)|^{2} d \omega, \quad t \geq 1
$$

The quantity $\sigma_{\mu}(t)$ plays the central role in restriction theory as well as the study of distance sets. See, for example, [W03] and the references contained therein for background. Let us point out one important geometric context where the quantity $\sigma_{\mu}(t)$ arises.

The work was partly supported by the National Science Foundation Grant DMS02-45369, a National Science Foundation Focused Research Grant, and an EPSRC grant GR/S13682/01.

AMS subject class 42B10 
The celebrated Falconer distance conjecture ([Fal86]) says that if the Hausdorff dimension of a compact set $E$ in $\mathbb{R}^{d}$ is greater than $\frac{d}{2}$, then the Lebesgue measure of the distance set $\Delta(E)$ is positive, with

$$
\Delta(E)=\{|x-y|: x, y \in E\},
$$

where $|\cdot|$ is the standard Euclidean distance. Wolff ([W99]) in dimension $d=2$ and Erdogan ([Erd05]) in all $d \geq 2$ proved that the Lebesgue measure of $\Delta(E)$ is indeed positive if the Hausdorff dimension of $E$ is greater than $\frac{d}{2}+\frac{1}{3}$. The proofs invoke highly non-trivial harmonic analysis, whereas a weaker exponent $\frac{d}{2}+\frac{1}{2}$ follows basically from the uncertainty principle, as shown by Sjölin ([Sj93]). The original approach to the Falconer distance problem was founded by Mattila ([Mat87]), aiming to prove that there exists a positive Borel measure $\mu$ supported on $E$ (with $\int d \mu>0$ ), such that

$$
M(\mu)=\int_{1}^{\infty} \sigma_{\mu}^{2}(t) t^{d-1} d t<\infty
$$

Then the Lebesgue measure of $\Delta(E)$ is positive. This is essentially the second moment argument, which is based on the fact that if $\nu$ is the (properly weighted) pull-forward of the measure $\mu \times \mu$ under the distance map $E \times E \mapsto \mathbb{R}$, then the one-dimensional Fourier transform

$$
\widehat{\nu}(t) \approx t^{\frac{d-1}{2}} \sigma_{\mu}(t)
$$

(The notations $\approx$, as well as further $\lesssim$ and $\gtrsim$ absorb constant multipliers - which may depend on the dimension and the diameter of the support of $\mu-$ in the usual way.) Hence, if (0.4) is true, the measure $\nu$ has an $L^{2}$ density, and its support cannot have zero Lebesgue measure, by the Cauchy-Schwartz inequality.

In order to vindicate (0.4), all of the aforementioned references sought to establish the estimate $\sigma_{\mu}^{2}(t) \lesssim t^{-\beta}$, for all $t \geq 1$ and some $\beta \leq s$, cf. (0.1). If this is the case, then from (0.4) - using polar coordinates and Plancherel - one has

$$
\begin{gathered}
M(\mu) \lesssim \int|\widehat{\mu}(\xi)|^{2}|\xi|^{-d+(d-\beta)} d \xi \\
\approx \iint|x-y|^{-(d-\beta)} d \mu(x) d \mu(y)=I_{d-\beta}(\mu),
\end{gathered}
$$$$
\text { the energy integral of } \mu \text { of order }-(d-\beta) \text {. This integral is bounded if } d-\beta \text { is smaller than }
$$
the Hausdorff dimension of $E$, or the quantity $s$.

In order to motivate the main result of this note we need a couple of pieces of notation. First, for a not necessarily positive (Schwartz class) function $f$, define

$$
\sigma_{f}(t)=\int_{S^{d-1}}|\widehat{f}(t \omega)|_{2}^{2} d \omega, \quad t \geq 1 .
$$


The spherical averages of Fourier transforms for general sign-indefinite measures have recently been addressed in a number of papers, see e.g. [SS99], [SS03] and the references contained therein.

Then define

$$
F I_{s}(f)=\int|\widehat{f}(\xi)|^{2}|\xi|^{-d+s} d \xi
$$

Observe that in the case when $f$ defines a positive measure in $\mathbb{R}^{d}$, the quantities $F I_{s}(f)$ and $I_{s}(f)$ are the same up to a constant, see [W03] for the detailed proof.

The following construction-based theorem indicates that without the positivity assumption, even though the "Fourier energy" integral $F I_{s}(f)$ is bounded, the Mattila integral $M(f)$ may behave quite badly, namely it will diverge in a range of $s>\frac{d}{2}$, satisfying the Falconer conjecture. This emphasizes the role of positivity in the context of the latter conjecture, even though the Fourier transform of $f$ in the construction exposed further turns out to be positive, as it is essentially the case in the lattice-based examples (see e.g [Fal96], [IR05]) which are characterized by similarity of both sides of the Fourier transform due to the applicability of the Poisson summation formula .

Theorem 0.1. For any $\frac{d}{2}<s<\frac{d^{2}}{2 d-1}$, there exists a one-parameter family $\mathcal{F}_{q}$ of $C^{\infty}$ functions supported in the unit cube, such that for any $f \in \mathcal{F}_{q}, \int\left|f_{q}(x)\right| d x \approx 1, F I_{s}\left(f_{q}\right) \approx 1$, while $M\left(f_{q}\right) \rightarrow \infty$ as $q \rightarrow \infty$.

We note that the assumptions of Theorem 0.1 are meant to mimic the positive case. More precisely, under the assumption that $f$ is a positive Borel measure, the condition $\int|f(x)| d x \approx 1$ says that $f$ is, approximately, a probability measure. The condition that $F I_{s}(f) \approx 1$ says in the positive measure context that $f$ is supported on a set of Hausdorff dimension $>s$. In particular, if we start out with a positive probability Borel measure $\mu$ supported on a set of positive Hausdorff dimension, then many reasonable localizations of $\mu$ on the Fourier transform side lead to functions satisfying the assumptions of Theorem 0.1. Our desire to understand the behavior of such functions in the context of the Falconer distance problems led us to the mathematics behind Theorem 0.1 .

If we are willing to relax the conditions in Theorem 0.1 a simpler construction ([T05]) can be used to yield a similar conclusion. Let $\widehat{f}(\xi)=|\xi|^{\frac{d-s}{2}} \phi\left(\xi-T \omega_{0}\right)$, where $\phi$ is a smooth cut-off function supported in the unit ball, $t$ is a large positive real parameter, and $\omega_{0}$ is a fixed vector of modulus 1 . Then

$$
\int|\widehat{f}(\xi)|^{2}|\xi|^{-d+s} d \xi \approx 1
$$

and

$$
\int_{S^{d-1}}|\widehat{f}(t \omega)|^{2} d \omega \approx T^{d-s} \cdot T^{-d+1} \chi_{[T-1, T+1]}(t)=T^{-s+1} \chi_{[T-1, T+1]}(t) .
$$


This leads immediately to the lower bound

$$
M(f) \geq \int_{T-1}^{T+1} T^{-2 s+2} \cdot t^{d-1} d t \approx T^{-2 s+d+1} \rightarrow \infty
$$

if $s \leq \frac{d+1}{2}$, a stronger conclusion than the one offered by Theorem 0.1. However, in this case

$$
|\widehat{f}(\xi)|=|\xi|^{\frac{d-s}{2}} \phi\left(\xi-T \omega_{0}\right) \geq T^{\frac{d-s}{2}}
$$

on the support of $\widehat{f}$, so $\int|f(x)| d x \rightarrow \infty$ as $T \rightarrow \infty$.

Acknowledgements. The authors wish to thank Burak Erdog̃an for several helpful remarks about the early draft of this paper. The authors also wish to thank Christoph Thiele for a helpful remark resulting in the comment following the statement of Theorem 0.1.

\section{SECTION 1: LOWER BOUND FOR $\sigma_{f}(t)$}

Let $q \gg 1$ and a function $f_{q}$ be defined by the relation

$$
\widehat{f}_{q}(\xi)=\phi\left(q^{-\frac{d}{s}} \xi\right) \sum_{a \in A} \phi(a q-\xi)
$$

where $\phi$ is a smooth radial cut-off function supported in the unit ball such that $\phi \geq 0$ and $A$ is a Delone set in the sense that elements of $A$ are $c$-separated, and any cube of side length $C$ in $\mathbb{R}^{d}$ contains some element of $A$, for some $0<c<C$. The function $f_{q}$ depends on the specific Delone set $A$ (to be described) and the large parameter $q$, which may be omitted in some notations. We shall need the following estimates.

Lemma 1.1. For every Delone set $A$, and all $q \gg 1$, the function $f_{q}$ is supported in the ball of radius 2 and

$$
\int\left|f_{q}(x)\right| d x \approx 1
$$

with constants independent of $q$.

Lemma 1.2. For every Delone set $A$,

$$
F I_{s}\left(f_{q}\right) \approx 1
$$

with constants independent of $q$. 
Let's take Lemma 1.1 and 1.2 for granted for the moment and obtain a lower bound for $\sigma_{f}(t)$. We have

$$
\left|\widehat{f}_{q}(\xi)\right|^{2} \approx\left|\widehat{\phi}\left(q^{-\frac{d}{s}} \xi\right)\right|^{2} \sum_{a \in A} \chi_{B_{\frac{1}{q}}(a)}(\xi / q)
$$

where $\chi_{B_{r}(x)}$ denotes the characteristic function of the ball of radius $r$ centered at $x$.

Since $\phi$ is radial, let $\phi_{0}$ be the function of one variable such that $\phi(\xi)=\phi_{0}(|\xi|)$. It follows that

$$
\begin{aligned}
\int_{S^{d-1}}\left|\widehat{f}_{q}(t \omega)\right|^{2} d \omega & \approx\left|\phi_{0}\left(q^{-\frac{d}{s}} t\right)\right|^{2} \sum_{a \in A} \int_{S^{d-1}} \chi_{B_{\frac{1}{q}}(a)}(t \omega / q) d \omega \\
& \approx\left|\phi_{0}\left(q^{-\frac{d}{s}} t\right)\right|^{2} \sum_{\frac{t}{q} \leq|a| \leq \frac{t}{q}+\frac{1}{q}} \frac{1}{t^{d-1}} .
\end{aligned}
$$

At this point, in order to get the desired family $\mathcal{F}_{q}$, we consider a special Delone set $A$ with specific properties we need. On each sphere of integer radius $m$ we place $\approx m^{d-1}$ 1-separated points of $A$. It follows that

$$
\sum_{\frac{t}{q} \leq|a| \leq \frac{t}{q}+\frac{1}{q}} 1 \approx\left(\frac{t}{q}\right)^{d-1}
$$

for a sequence of $t$ s going to infinity, when we have

$$
\int_{S^{d-1}}\left|\widehat{f}_{q}(t \omega)\right|^{2} d \omega \gtrsim\left|\phi_{0}\left(q^{-\frac{d}{s}} t\right)\right|^{2} \frac{1}{q^{d-1}} .
$$

Observe that choosing $q \approx t^{\frac{s}{d}}$, as we may, we obtain

$$
\int_{S^{d-1}}\left|\widehat{f}_{q}(t \omega)\right|^{2} d \omega \gtrsim t^{-s+\frac{s}{d}}
$$

for a sequence of $t$ s going to infinity (and a sequence of members of the family $\mathcal{F}_{q}$ ).

Section 2: Proof of Lemma 1.1 And Lemma 1.2

To prove that $f_{q}$ in Lemma 1.1 is compactly supported, we write

$$
f_{q}(x)=\sum_{a \in A} \int e^{2 \pi i x \cdot \xi} \widehat{\phi}\left(q^{-\frac{d}{s}} \xi\right) \widehat{\phi}(a q-\xi) d \xi
$$




$$
\begin{gathered}
=\sum_{a \in A} \iint e^{2 \pi i(x-y) \cdot \xi} \widehat{\phi}\left(q^{-\frac{d}{s}} \xi\right) d \xi e^{2 \pi i q a \cdot y} \phi(y) d y \\
=q^{\frac{d^{2}}{s}} \sum_{a \in A} \int e^{2 \pi i q a \cdot y} \phi\left(q^{\frac{d}{s}}(x-y)\right) \phi(y) d y,
\end{gathered}
$$

from which it is apparent that $f$ vanishes identically outside the ball of radius 2 . The validity of (1.2), i.e. that $\left|\widehat{f}_{q}(\xi)\right| \lesssim 1$ is obvious by definition of $f_{q}$.

To prove Lemma 1.2, observe that

$$
\left|\widehat{f}_{q}(\xi)\right|^{2} \approx\left|\phi\left(q^{-\frac{d}{s}} \xi\right)\right|^{2} \sum_{a \in A} \chi_{B_{\frac{1}{q}}(a)}(\xi / q) .
$$

It follows that

$$
\begin{gathered}
F I_{s}\left(f_{q}\right)=\int\left|\widehat{f}_{q}(\xi)\right|^{2}|\xi|^{-d+s} d \xi \approx \sum_{a \in A} \int\left|\widehat{\phi}\left(q^{-\frac{d}{s}} \xi\right)\right|^{2} \chi_{B_{\frac{1}{q}}(a)}(\xi / q)|\xi|^{-d+s} d \xi \\
=\int\left|\widehat{\phi}\left(q^{-\frac{d}{s}} \xi\right)\right|^{2} \chi_{B_{\frac{1}{q}}(0, \ldots, 0)}(\xi / q)|\xi|^{-d+s} d \xi \\
+\sum_{a \neq(0, \ldots, 0)} \int\left|\widehat{\phi}\left(q^{-\frac{d}{s}} \xi\right)\right|^{2} \chi_{B_{\frac{1}{q}}(a)}(\xi / q)|\xi|^{-d+s} d \xi=I+I I .
\end{gathered}
$$

Clearly, $I \approx 1$. On the other hand,

$$
\begin{aligned}
I I & \approx C_{N} q^{-d+s} \sum_{a \neq(0, \ldots, 0)}|a|^{-d+s} \int\left(1+q^{-\frac{d}{s}}|\xi|\right)^{-N} \chi_{B_{\frac{1}{q}}(a)}(\xi / q) d \xi \\
& =C_{N} q^{s} \sum_{a \neq(0, \ldots, 0)}|a|^{-d+s} \int\left(1+q^{-\frac{d}{s}+1}|\xi|\right)^{-N} \chi_{B_{\frac{1}{q}}(a)}(\xi) d \xi \\
& \approx q^{s} q^{-d} \sum_{1 \leq|a| \leq q^{\frac{d}{s}-1}}|a|^{-d+s} \approx q^{s} q^{-d}\left(q^{\frac{d}{s}-1}\right)^{s} \approx 1,
\end{aligned}
$$

and the proof of Lemma 1.2 is complete. 


\section{Section 3: Proof of Theorem 0.1: the Conclusion}

We must estimate

$$
\int_{1}^{\infty}\left(\int_{S^{d-1}}\left|\widehat{f}_{q}(t \omega)\right|^{2} d \omega\right)^{2} t^{d-1} d t
$$

¿From (1.8) and (1.9) we know that

$$
\int_{S^{d-1}}\left|\widehat{f}_{q}(t \omega)\right|^{2} d \omega \gtrsim \frac{1}{q^{d-1}}
$$

whenever $\frac{t}{q}$ is an integer, and the estimate persists as $t$ varies from $m q$ to $m q+1$. It follows that

$$
\begin{aligned}
& M(f) \gtrsim \sum_{m=1}^{q^{\frac{d}{s}-1}} \int_{m q}^{(m+1) q}\left(\int_{S^{d-1}}\left|\widehat{f}_{q}(t \omega)\right|^{2} d \omega\right)^{2} t^{d-1} d t \\
& \gtrsim \sum_{m=1}^{q^{\frac{d}{s}-1}} \frac{1}{q^{2(d-1)}}(m q)^{d-1} \gtrsim \frac{1}{q^{d-1}} q^{\frac{d^{2}}{s}-d}=q^{\frac{d^{2}}{s}-2 d+1} .
\end{aligned}
$$

The power of $q$ on the right hand side of (3.3) is positive unless

$$
s \geq \frac{d^{2}}{2 d-1}
$$

as claimed. This completes the proof of Theorem 0.1 (the support in the ball of radius two claimed by Lemma 1.1 can be transformed into the unit cube as stated in the Theorem by straight-forward scaling). 


\section{REFERENCES}

[Erd05] B. Erdog̃an, A bilinear Fourier extension theorem and applications to the distance set problem, IMRN (accepted for publication) (2005).

[Fal86] K. J. Falconer, On the Hausdorff dimensions of distance sets, Mathematika 32 (1986), 206-212.

[IR05] A. Iosevich and M. Rudnev, Spherical averages, lower bounds, and lattice points on convex surfaces, (submitted for publication) (2005).

[Mat87] P. Mattila, Spherical averages of Fourier transforms of measures with finite energy: dimensions of intersections and distance sets, Mathematika 34 (1987), 207-228.

[Sj93] P. Sjölin, Estimates of spherical averages of Fourier transforms and dimensions of sets, Mathematika 40 (1993), 322-330.

[SS99] P. Sjölin, F. Soria, Some remarks on restriction of the Fourier transform for general measures, Publ. Mat. 43 (1999, no. 2,), 655-664.

[SS03] P. Sjölin, F. Soria, Estimates of averages of Fourier transforms with respect to general measures, Proc. Roy. Soc. Edinburgh Sect. A 13 (2003 no. 4,), 943-950.

[T05] C. Thiele, (Personal communcation) (2005).

[W99] T. Wolff, Decay of circular means of Fourier transforms of measures, International Mathematics Research Notices 10 (1999), 547-567.

[W03] T. Wolff, Lecture notes on harmonic analysis. With a foreword by Charles Fefferman and preface by Izabella Eaba. Edited by Eaba and Carol Shubin., University Lecture Series, 29. American Mathematical Society, Providence, RI, (2003).

Department of Mathematics, University of Missouri-Columbia, Columbia Missouri 65211 USA

E-mail address: iosevich @ math.missouri.edu

Department of Mathematics, University of Bristol, Bristol BS8 1TW, UK

E-mail address: m.rudnev @ bristol.ac.uk 